\title{
Marxisme et critique littéraire
}

Lucile Dumont, Quentin Fondu, Laélia Véron

\section{Introduction}

Ni Marx ni Engels, non plus que Paul Lafargue, Lénine ou Trotski, n’ont proposé de théorie de la littérature. Leurs analyses littéraires ont pris la forme d'écrits de circonstances, sans réflexions approfondies sur la spécificité de la littérature ou sur son statut idéologique. L'absence de corpus consacré aux objets littéraires parmi les textes classiques du communisme a pendant longtemps empêché l'apparition d'une critique littéraire marxiste relativement indépendante des enjeux politiques.

À partir de la Libération, les approches marxistes de la littérature se développent pourtant et s'intéressent, sous un angle matérialiste, aux déterminations sociales de la production littéraire, contre les traditions idéalistes dominantes. Le contrôle initial du Parti communiste français $(\mathrm{PCF})$ sur les corpus marxistes puis sur leur diffusion se relâche à partir du milieu des années 1950, à la faveur de la crise internationale ouverte par le XXème Congrès du PCUS qui amorce la déstalinisation. Au même moment, les références au marxisme se multiplient dans les domaines culturel et intellectuel. La critique littéraire revendiquant un marxisme hétérodoxe, souvent issue de membres de la gauche antistalinienne, réinvestit alors la question des formes, contre l'idée que les productions artistiques ne seraient que le « reflet» de leurs conditions matérielles de production. Il s'agit également, pour certains d'entre eux, de rendre visibles les mécanismes de pouvoir véhiculés par les discours grâce à la pratique de la critique de l'idéologie. Dans le contexte des transformations de la critique littéraire française, les appropriations du marxisme se diversifient et recouvrent peu à peu des pratiques intellectuelles et des positionnements politiques hétérogènes. A partir des années 1970, si certains universitaires prolongeant la construction d'approches marxistes trouvent une place dans l'université, la revendication explicite du marxisme décline.

\section{L'introduction du marxisme dans la critique littéraire (Années 50)}

\section{Le Parti communiste français face à la question littéraire}

L'intérêt du PCF pour la littérature ne date certes pas de 1945, comme le prouve l'existence, durant l'entre-deux guerres, d'une critique littéraire communiste dans plusieurs journaux (en particulier L'Humanité), ou encore le rôle accordé aux écrivains et aux artistes dans la lutte 
antifasciste. Le PCF oscille alors entre la promotion d'auteurs considérés comme progressistes (majoritairement français) et l'usage politique des enjeux littéraires. Ce n'est qu'après la Libération qu'il développe une approche spécifique de la littérature. En décalage chronologique avec la politique culturelle et intellectuelle de l'URSS, les orientations du PCF témoignent d'une prise en compte tardive des enjeux culturels officiels. Si le réalisme socialiste, doctrine culturelle de l'URSS promouvant une représentation figurative et héroïque du peuple, avait été imposé contre les avant-gardes artistiques par Andreï Jdanov dès 1934, ce n'est qu'en 1947 que le PCF l'adopte, à la suite d'un durcissement idéologique qui, tout comme en URSS, marque le début de la Guerre froide. Si le réalisme socialiste représente à la fois une « méthode de création et $[\ldots]$ une catégorie de critique » (Aron, Matonti, Sapiro, 2003 : 167), ses principes sont multiples et peuvent faire référence soit à une « définition esthétique rigoureuse [qui] en rétrécit singulièrement le domaine », soit à une « définition large [qui] le réduit en fin de compte à un critère idéologique et politique sans véritable contenu esthétique » (Aucouturier, 1998 : 110). Sa définition est ainsi un objet de conflit. Pour Georg Lukács et ses adeptes, le réalisme français du XIXème siècle représente un modèle à suivre. Roger Garaudy, dans ses prises de position orthodoxes au sein du PCF, refuse de réduire le réalisme socialiste à une simple esthétique formelle au profit d'une conception idéologique de l'art, fidèle aux principes du jdanovisme exigeant de faire de la politique un «guide» (Jdanov, 1950:25). À partir de D’un réalisme sans rivages (1963), sa position se détourne de cette conception idéologique et évolue vers une «métaphysique de la création ("l’œuvre artistique comme projection dans l'avenir du possible humain") » («Littérature », in Dictionnaire critique du marxisme, 2001 : 662). Enfin, le débat qui oppose Francis Jourdain, artiste-peintre, et André Wurmser au sein de la revue La Pensée en 1950 montre bien que la définition du rôle du marxisme et du PCF en matière artistique continue à opposer une conception « grossière » (au détriment de la liberté en art) et une conception «modeste» (au détriment de la conception politique de l'art) : «Je suis donc heureux que vous me félicitiez de m'être élevé contre ce "marxisme grossier qui se laisserait réduire à une table de logarithmes". Permettez-moi cependant de ne pas lui préférer ce marxisme par trop modeste qui, frappant à la porte de l'atelier, s'excuserait auprès de sa Majesté l'artiste : "Oh ! Pardon, je me suis trompé d'étage..." et redescendrait chez le cordonnier, bien décidé de ne jamais monter plus haut que la chaussure. »(Wurmser, 1950 : 133.)

Obéissant à une stratégie d'investissement des espaces intellectuels, la politique d'édition d'auteurs marxistes du PCF prend de l'importance à cette même époque. Les Éditions sociales proposent ainsi des traductions ou des rééditions d'ouvrages d'auteurs marxistes consacrés à la littérature, accompagnées de longues présentations signées par Jean Fréville (1895-1971). C'est 
le cas de la réédition de l'anthologie des textes de Marx et d'Engels sur la littérature et l'art (1954, préface de Maurice Thorez), de L'Art et la vie sociale (1950) de Gueorgui Plekhanov ou encore d'un choix de texte de Lénine, Sur la littérature et l'art (1957). Il s'agit pour le PCF de constituer un canon marxiste d'interprétation des objets littéraires et artistiques, ainsi que d'inscrire le réalisme socialiste dans l'histoire du marxisme d'une part et dans la tradition nationale française d'autre part, comme le montre la préface de Maurice Thorez aux textes de Marx et d'Engels où il écrit « qu'avec le premier recueil : Sur la littérature et l'art, le marxisme a fait irruption dans des domaines que se réservaient jalousement les coryphées de la critique bourgeoise. [...] La pensée marxiste donne des bases théoriques à un réalisme axé sur le progrès social et la transformation du monde. » (Marx, Engels, 1954 : 10.)

\section{La fin d'un monopole?}

Si le PCF investit de plus en plus les espaces intellectuels à la suite de la Libération, c'est aussi parce qu'il est concurrencé sur ce terrain par les intellectuels qui n'en sont pas membres et qui s'intéressent de plus en plus au marxisme. Ainsi, contre la prétention au monopole du PCF, certains d'entre eux, Jean-Paul Sartre en tête, refusent l'étiquette " marxiste » tout en partageant certains de ses principes. Dans Qu'est-ce que la littérature? (1948), Sartre s'oppose à la critique littéraire traditionnelle et formule sa théorie de la responsabilité de l'écrivain, qu'il conçoit comme forme nouvelle d'engagement intellectuel et artistique détaché des enjeux partisans. Auteur engagé et promoteur d'une critique matérialiste de la littérature, qu'il mettra en œuvre dans L'Idiot de la famille (1971-1972) consacré à Flaubert, Sartre entretient tout au long de sa carrière des rapports ambigus au marxisme de même qu'avec le parti communiste.

On assiste au même moment à l'importation en France des premiers travaux de Georg Lukács, au sein des intellectuels de gauche critiques du stalinisme regroupés autour de la revue Arguments (1956-1962). La circulation de ses écrits doit beaucoup à Lucien Goldmann (19131970), qui consacre au théoricien hongrois un long texte, «Introduction aux premiers écrits de Georg Lukács », publié dans Les Temps modernes en 1962. Contre ceux qu'il nomme les théoriciens marxistes «du reflet» (Gueorgui Plekhanov et Franz Mehring en particulier) et contre la sociologie de la littérature empirique qui s'établit autour de Robert Escarpit (19182000), Lucien Goldmann construit une épistémologie et une méthodologie singulières, attentives aux structures sociales et à leurs transformations. Ce «structuralisme génétique » constitue les fondements de sa sociologie de la littérature : il s'oppose aussi bien à la réduction de l'œuvre à son contexte de production, que promeut selon lui le marxisme mécaniste, qu'à sa réduction à la biographie de son auteur, mobilisée dans l'enseignement littéraire. Dans Le Dieu 
caché (1955), il montre que, pour être comprises, les œuvres de Pascal comme celles de Racine doivent être rattachées à une « vision du monde », « cet ensemble d'aspirations, de sentiments et d'idées qui réunit les membres d'un groupe (le plus souvent, d'une classe sociale), et les oppose aux autres groupes. »(Goldmann, 2013 : 349) Cette vision tragique, qui est celle du groupe social de la noblesse de robe déclassée brutalement par le pouvoir royal, est une vision du monde paradoxale et sans possibilité de transformations - sans histoire - que l'on retrouve à la fois dans la théologie janséniste et dans les structures des œuvres de Racine et de Pascal, où Dieu est à la fois omniprésent et absent. La relecture des ouvrages du jeunes Lukács, ainsi que le choix d'un corpus prestigieux et de l'analyse marxiste des textes constituent alors un double positionnement : par rapport à la ligne politique du PCF et par rapport à la critique littéraire dominante, en revendiquant simultanément la nécessité de prendre en compte le contexte de production des œuvres, y compris pour les œuvres classiques, de même que leur irréductibilité.

\section{La promotion d'un marxisme hétérodoxe : le cas Brecht}

Dans un moment de discrédit du PCF qui fait suite à son soutien à l'intervention soviétique en Hongrie en 1956, la réception de Brecht en France participe également de la promotion d'un marxisme hétérodoxe. Peu connu avant-guerre, ce n'est que dans les années 1950 que Brecht triomphe sur les scènes françaises. Alors qu'il est jugé trop formaliste et trop éloigné des canons du réalisme socialiste par le PCF, il représente, à l'inverse, la possibilité d'une «réflexion marxiste mais par des voies brechtiennes », comme l'écrira Bernard Dort, critique théâtral. Dans le but d'allier plaisir de la représentation théâtrale et rôle pédagogique du théâtre, Brecht formule sa théorie de la «distanciation » par laquelle il refuse l'illusion théâtrale de la scène à l'italienne, ainsi que l'identification des spectateurs aux personnages. À propos de Mère Courage par exemple, qui narre l'aveuglement d'une mère face à la guerre, le dramaturge écrit que «le spectateur ne doit pas vivre ce que vivent les personnages, mais mettre ceux-ci en question ». Il propose par conséquent une nouvelle théorie de l'engagement artistique ayant pour projet de déconstruire l'idéologie dominante plutôt que de faire œuvre de propagande. Le projet esthético-politique brechtien est introduit et soutenu en France par Roland Barthes qui, dans Mythologies (1957), se donne pour projet de traquer les idéologies en observant, dans les objets et les discours quotidiens, « la façon dont un lexique et une grammaire peuvent être politiquement engagés »(Barthes, 1957 : 777). À la fois théoricien et artiste, Brecht peut ainsi représenter au cours de cette période un «modèle » tant esthétique que 
politique, tandis que (le vieux) Lukács, par son accommodement au stalinisme, agit comme « repoussoir » (Matonti, $2005:$ 174).

Ces deux formes d'hétérodoxie à l'égard de l'orthodoxie communiste, la première, dans la filiation des travaux du jeune Lukács, refusant de sacrifier les œuvres sur l'autel des déterminations sociales et la seconde optant pour une critique non-idéologique de l'idéologie à la suite de Brecht, partagent une même opposition à la «théorie du reflet », étiquette négative accolée à la critique mobilisée dans l'orbite du PCF (d'ailleurs lui-même en voie de renouvellement).

\section{De la théorie marxiste aux théories littéraires (années 1960 -1970)}

\section{Marxisme, communisme et structuralisme littéraire}

À la suite du processus de déstalinisation, une série de transformations marque l'évolution du PCF dans les années 1960 et favorise la diffusion et l'appropriation de différentes orientations marxistes en matière de critique littéraire. Entre nécessité du renouvellement intellectuel et crainte face à l'ouverture politique, les déchirements des communistes se lisent également dans leur traitement de la littérature. En 1960 est créé le Centre d'Études et de recherches marxistes (CERM), qui publie la Bibliographie marxiste internationale à partir de 1964. Dans le même temps, l'essor des sciences humaines est marqué par le développement des approches structuralistes de la culture et plus spécifiquement de la littérature. Celles-ci sont très tôt questionnées sur leur rapport au marxisme. Le centre de Cerisy-la-Salle, lieu d'un marxisme «politiquement anti-stalinien, non léniniste et aussi non maoïste, et intellectuellement antialthussérien » (Jeanpierre 2013) accueille en 1959 l'un des premiers colloques consacrés au structuralisme, «Genèse et structure », organisé conjointement par Maurice de Gandillac, Lucien Goldmann et Jean Piaget. Quelques années plus tard, c'est l'ethnologue Lucien Sebag qui, dans Marxisme et structuralisme (1964), interroge les possibles continuités entre ces deux approches. Le dialogue entre marxisme et structuralisme change progressivement de forme au cours des années 1960 avec l'émergence du structuralisme dans sa version althussérienne, qui promeut un marxisme scientifique en rupture avec l'héritage de la pensée de Lukács. L'importation des formalistes russes, initialement cadrée par les réseaux du PCF, contribue à cette transition. L'appropriation française des travaux formalistes, dont les représentants étaient opposés à la critique marxiste-léniniste soviétique, servent autant la critique du jdanovisme que le renouvellement de la critique littéraire et de l'esthétique (Depretto 2010). 
Dès lors, si Aragon s'était prononcé dès 1959 en faveur de l'abandon du réalisme socialiste, les intellectuels communistes les plus autonomes prennent peu à peu part à la constitution et à la promotion de leur «propre théorie de la littérature, voie médiane entre la théorie du reflet et le formalisme telquélien » (Matonti 2005 : 197-198), qui s'élabore à distance d'Aragon comme de Garaudy. La doctrine du réalisme socialiste n'est pourtant officiellement abandonnée qu'en 1966 lors du Comité central d’Argenteuil (Martelli 2017). Celui-ci est également un moment de diffusion et de discussion des travaux d'Althusser, dont les travaux ne tardent pas à nourrir les théories littéraires. Proches des disciplines nouvelles (sémiologie, linguistique...), elles rencontrent un succès important dans les espaces académiques, intellectuels et littéraires alors fortement politisés. La multiplication des revues, la croissance des lettres modernes dans les universités, la montée du structuralisme et la contestation du modèle traditionnel des études littéraires sont en outre à l'origine d'une transformation globale de la critique littéraire, dans laquelle les travaux d'inspiration marxiste occupent une place grandissante et essentielle pour la formalisation théorique. L'organisation d'un débat public à la Mutualité en février 1965 par l'Union des Étudiants Communistes (UEC) et son journal Clarté, tous deux critiques du jdanovisme, sur le thème «Que peut la littérature ?» témoigne de cet investissement des questions littéraires et des divisions à l'intérieur du marxisme. Le débat oppose Jean-Pierre Faye et Jean Ricardou, alors promoteurs des approches théoriques de la littérature, à Sartre et Simone de Beauvoir qui défendent leur vision de la littérature engagée, tandis que Jorge Semprún tente de (re)faire de la littérature un levier révolutionnaire.

$\mathrm{Au}$ sein du renouveau théorique de la critique littéraire, ce sont des appropriations hétérodoxes du marxisme qui dominent. La trajectoire de Roland Barthes (1915-1980), l'une des principales figures des approches théoriques de la littérature, est ainsi placée sous le signe d'un marxisme dissident et d'un « recodage » du lexique marxiste qui témoigne autant de son rattachement au marxisme que de son refus de s'y rallier pleinement (Roger 1996). Jamais adhérent du PCF, Barthes a conservé une distance critique, tout au long de sa carrière, à l'égard du communisme et de ses figures littéraires. Dès son premier ouvrage, Le Degré zéro de l'écriture (1953), il avait pris Garaudy et André Stil pour cible. Barthes envisageait le marxisme comme une méthode de laquelle il ne s'est revendiqué que rétrospectivement. La critique de l'idéologie, associée à la sémiologie, fut une manière de faire le procès de la «critique universitaire » à laquelle il s'est opposé, et de proposer des analyses littéraires, comme celle de l'effet de réel. La - lointaine - participation de Barthes à Arguments, sa défense du théâtre de 
Brecht et sa proximité ponctuelle avec Goldmann l'ont également ancré dans des réseaux marqués par des usages hétérodoxes du marxisme.

\section{« Pratique théorique » et renouvellement des études littéraires.}

Les revues Tel Quel et La Nouvelle Critique sont à l'origine de deux colloques tenus à l'abbaye de Cluny en 1968 et 1970, respectivement intitulés « Littérature et linguistique » et «Littérature et idéologies ». Lors de ce second colloque, particulièrement houleux aux dires de ses organisateurs, certains textes canoniques auparavant monopolisés par le PCF, ceux de Lénine en particulier, font l'objet d'appropriations conflictuelles. Parmi les collaborateurs réguliers à Tel Quel, Julia Kristeva (1941-), née en Bulgarie et élève de Goldmann et de Barthes, propose une approche de la littérature basée sur une alliance de la sémiologie avec un althussérisme qui marque la revue jusqu'au début des années 1970. La notion d' « intertextualité » proposée par Kristeva dès le milieu des années 1960, qui consiste à déceler l'ensemble des textes mis en relation dans un texte donné, formalise la critique de l'idéologie en la dotant d'outils d'analyse linguistique qui permettent de revendiquer une assise scientifique. Dans le même temps, elle permet de distinguer les caractéristiques du langage littéraire et de les distinguer des usages « ordinaires » de la langue, avec lesquels la littérature se trouve toutefois dans un rapport de continuité. La question du rapport entre le discours littéraire et les usages courants de la langue sera examinée plus tard par la linguiste Renée Balibar, également dans un cadre d'analyse marxiste proche des conceptions althussériennes (Les français fictifs. Le rapport des styles littéraires au français national, 1974).

L'application du marxisme althussérien à la littérature est aussi au fondement du premier ouvrage de Pierre Macherey, Pour une théorie de la production littéraire, publié en 1966 chez Maspero. L'élève d'Althusser y présente une approche de la littérature qui promeut la « pratique théorique » marxiste comme le véhicule d'un discours scientifique sur la littérature. Celui-ci n'est possible, selon Macherey, qu'au prix d'une rupture avec la pratique de la littérature de même qu'avec le jugement esthétique. La réflexion sur les conditions de l'autonomie de l'écrivain, la prise en compte de la dimension sociale de la « production » littéraire - terme qu'il substitue à celui de «littérature »- qui fait de l'écrivain l'ouvrier de son texte, ainsi que la nécessité d'introduire des médiations qui contribuent à la production littéraire sont des axes majeurs de ce travail. Il y discute les textes de Lénine sur Tolstoï, dont il critique la dimension 
strictement politique, qui lui permet d'interroger les conditions dans lesquelles est produit un discours littéraire. Il réfute les théories du reflet et les approches herméneutiques et structurales de la littérature. Il envisage cette dernière comme un discours spécifique qui ne peut être compris que de manière relationnelle et qui, surtout, recèle un pouvoir critique tel qu'elle devient un instrument privilégié de la critique de l'idéologie. Au même moment, les Cahiers Marxistes-Léninistes, créés en 1964 à l'initiative du groupe de l'UEC de la rue d'Ulm font la promotion de conceptions similaires. Le numéro «Pouvoirs de la littérature » (1965-1966) témoigne de l'importance des questions littéraires dans ce groupe, qui apparaissent aussi comme un objet propice à la mise en débat de différentes conceptions du marxisme. L'article ironiquement intitulé « Monsieur Goldmann bouleverse la science » dans le $n^{\circ} 3$ de la revue dit bien les fractures internes aux approches marxistes des objets littéraires. En reprochant à Goldmann la « dénaturation » de ses analyses de Marx, en particulier sa lecture de la réification, les auteurs affirment que son travail est d'ordre idéologique et se trouve par conséquent dans l'impossibilité de devenir scientifique.

La crise de mai 68 est un moment d'intensification de la production théorique et une matrice de radicalisation politique, qui finit cependant par l'éclatement des groupes théoriques et la délégitimation progressive de Tel Quel (Gobille 2005). Barthes ne se montre pas indifférent aux approches althussériennes et affiche son soutien à Sollers et son équipe. Deux de ses élèves, Gérard Genette (1930-) et Tzvetan Todorov (1939-2017), sont à l'origine de théories de la littérature qui systématisent les méthodes structurales d'analyse littéraire autour de la revue académique Poétique (1970). Celle-ci rencontre un important succès dans l'enseignement littéraire et éloigne les théories de la littérature des réseaux marqués par la diffusion du marxisme dans lesquels elles étaient initialement inscrites, même dans leur version en apparence la moins politisée - Genette avait été membre de Socialisme ou Barbarie et Todorov, qui avait été très tôt familiarisé aux écrits de dissidents des régimes communistes, était l'un des importateurs principaux des formalistes russes. Enfin, la publication de la monumentale Histoire littéraire de la France entre 1974 et 1980 par les Éditions Sociales, la maison d'édition historique du PCF, montre que les instances culturelles et intellectuelles du parti ne cessent pas pour autant d'investir le terrain de la littérature. Réunissant des collaborateurs d'horizons variés, la collection de 12 volumes affirme, dans la lignée des approches théoriques, la nécessité de rompre avec l'histoire littéraire biographique tout en affirmant la volonté de « ne pas séparer l'histoire de la littérature de l'histoire tout court » (Desné 1974 :9) et par là de rendre à l'histoire littéraire sa dimension sociale et politique. 


\section{Mutations et déclin du marxisme dans les approches littéraires}

La revendication du marxisme comme doctrine globale s'estompe progressivement à partir du milieu des années 1970. La perte d'influence du PCF dans le monde universitaire ne s'accompagne pas de nouvelles formes d'institutionnalisation des approches marxistes de la littérature (à l'exception, limitée dans le cas des études littéraires, de Vincennes). Si les références au marxisme ne disparaissent pas dans les études littéraires françaises, elles se transforment : à la vogue théorique des années 1960 succède peu à peu une approche plus empirique et moins doctrinaire du marxisme. L'article de Pierre Macherey et d'Étienne Balibar «Sur la littérature comme forme idéologique » (paru dans la revue Littérature en 1975), qui prétend établir une théorie des effets littéraires, apparaît plus comme un chant du cygne althussérien que comme le signe d'un renouveau théorique. La réception, favorable, mais ponctuelle, de figures littéraires marxistes à l'université (parmi lesquelles Pierre Barbéris ou encore Roger Fayolle), la naissance d'une discipline, la sociocritique (marquée, à l'origine, par une sensibilité marxiste, mais qui s'élabore sur de nouveaux concepts), l'essor de la sociologie de la littérature, manifestent tout à la fois un intérêt, souvent éclectique, pour des méthodes d'inspiration marxiste et un abandon de la théorie marxiste comme système.

\section{Barbéris ou l'incarnation d'un marxisme de chaire}

L'une des figures majeures d'une conception marxiste du domaine et des méthodes de la critique littéraire à l'intérieur même des études littéraires est Pierre Barbéris (1926-2014), enseignant, chercheur, mais aussi militant politique et syndical. Auteur d'une œuvre considérable, il devient une figure reconnue dans le monde universitaire, malgré des heurts avec la Sorbonne ou avec des critiques, comme Bernard Guyon, qui lui reprochent une approche doctrinaire. Si bon nombre de chercheurs en littérature témoignent, à l'époque, d'une sensibilité marxisante, Barbéris est un des rares à se proclamer, tout au long de sa carrière, critique marxiste. Il entend injecter une conception marxiste affinée de l'histoire dans l'approche des textes littéraires et, pour ce faire, s'écarte aussi bien d'une certaine orthodoxie marxiste (qu'il s'agisse d'un Lukács trop philosophe à son goût ou du théoricisime d'Althusser) que d'une vision positiviste ou idéaliste dominante dans les études littéraires. Les grands ouvrages de Barbéris sont principalement des lectures de textes classiques (Balzac, Stendhal), fondées sur des principes marxistes, qui replacent les auteurs étudiés par rapport à l'éclosion de la pensée marxiste et à l'horizon de la lutte des classes. La position institutionnelle de Barbéris, à la fois 
reconnue et limitée (il est professeur à l'ÉNS de Lyon, puis à l'université de Caen) permet de prolonger quelque peu l'imprégnation marxiste de la discipline. Cependant, si ses lectures enthousiasment une génération d'élèves (Gérard Gengembre, Jean Goldzinck, Nicole Mozet), elle ne donne pas lieu à des prolongements institutionnels. Barbéris apparaît ainsi comme le butte-témoin d'une époque où marxisme et recherche littéraire étaient assez étroitement liés.

Cette reconnaissance institutionnelle dont a pu jouir Barbéris le différencie d'André Wurmser, journaliste et écrivain français, éditorialiste à L'Humanité. S'ils se réclament tous deux du marxisme, Barbéris mène une carrière académique classique alors que la somme critique de Wurmser sur Balzac, La Comédie inhumaine, est généralement raillée par les spécialistes. Barbéris prendra d'ailleurs ses distances avec Wurmser dont il critiquera l'étiquette marxiste comme purement « politicienne » (Post-face du Monde de Balzac, 2000).

\section{Le développement d'une sociologie non marxiste du champ littéraire : l'exemple de}

\section{Bourdieu}

La sociologie de la littérature, qui étudie la littérature (qu'il s'agisse de production ou de réception de la littérature) en tant que fait social, s'est détachée peu à peu d'une approche marxiste, telle qu'elle a été promue par Lucien Goldmann à la fin des années 1960. Cette sociologie marxiste a été mise à mal aussi bien par le succès des critiques immanentistes que par la difficulté éprouvée par Goldmann lui-même à faire évoluer son approche critique tout en restant fidèle aux principes théoriques marxistes. Ainsi dans Pour une sociologie du roman (1964), Goldmann substitue-t-il la notion de groupe social à celle de classe sociale, ce qui lui vaudra d'être taxé de déviationnisme anti-marxiste. Après le décès brutal de Goldmann en 1970, cette sociologie ne dispose d'aucun ancrage institutionnel fort et ne parvient à faire école ni chez les littéraires ni chez les sociologues. Le souhait de Goldmann d'établir comme discipline une sociologie marxiste de la littérature ne se réalisera donc pas.

Parmi les nouvelles approches sociologiques de la littérature, on peut citer celle de Pierre Bourdieu (1930-2002), qui reprend certains concepts marxistes comme ceux de classe ou de capital, mais dans une perspective théorique et méthodologique différente. D’une part, en établissant la spécificité d'un champ littéraire régi par des contraintes propres, Bourdieu dessine un espace social complexe et fragmenté, loin de la traditionnelle philosophie de l'histoire marxiste (certains marxistes lui reprochant ainsi de ne pas préciser la façon dont ces champs s'agencent les uns avec les autres, et ainsi de ne pas proposer de théorie générale de la société). Bourdieu (1984) déclare refuser aussi bien Hegel que Marx (auquel il associe Lukács et Goldmann), c'est-à-dire aussi bien l'autonomie des œuvres qu'une relation directe de l'œuvre 
et du social. D'autre part, la méthode d'analyse du champ littéraire explicitée par Bourdieu (1984) (situation du champ littéraire au sein du champ du pouvoir, analyse interne du champ littéraire, analyse de l'habitus de l'écrivain) vise à comprendre des trajectoires d'auteur mais sans, comme Goldmann, les rattacher à l'expression d'une situation existentielle transindividuelle - «l'homologie entre les deux types de structures - romanesque et sociale éta[nt] conditionnée par la socialisation de l'écrivain et par l'habitus qui en résulte (et non pas, comme chez Goldmann, par la vision du monde de son groupe social) (Dirkx, 2015).

\section{L’ambiguïté de la sociocritique}

Dans le contexte idéologique et politique post-mai 68 , la sociocritique, introduite à l'Université de Vincennes par Claude Duchet en 1971, apparaît «comme une dissidence théorique de l'approche marxiste traditionnelle » (Laforgue, 2003). Dans son article-manifeste, Duchet récuse tout à la fois les lectures poétiques qui excluent le social du texte et le traitement du social par l'analyse littéraire marxiste, figée selon lui par « la théorie du reflet, le concept de typique (...), une insuffisante exploration des idéologies et de la nature du signifié littéraire » (1971), cherchant ainsi à délimiter une nouvelle approche non pas du texte littéraire comme fait social, comme la sociologie de la littérature, mais du social dans le texte littéraire. Un nouvel espace disciplinaire s'ouvre, espace dont les contours ne sont pas nettement définis, qui devient l'objet de luttes symboliques et de débats théoriques, dont le marxisme sort perdant à la fin du siècle.

En effet, le mot sociocritique est rapidement victime de son succès : revendiqué dans des optiques très différentes, il peine à désigner une approche précise. Cet embarras est résumé par Henri Mitterrand lors de la clôture du colloque de Toronto en 1972 sur la sociocritique du texte romanesque : «Nous n'avons pas réussi (...) à dégager une définition de la sociocritique, ni même une claire conscience de ses objectifs, de ses perspectives et de ses langages. »Cet embarras est également visible dans la préface de Sociocritique (1979), dans laquelle Claude Duchet cherche à la fois à rendre hommage à la sociologie de la littérature, à la critique matérialiste marxiste (et notamment à Lucien Goldmann) et à s'en distinguer, en prônant une « réorientation de l'investigation socio-historique du dehors vers le dedans, c'est-à-dire l'organisation interne des textes, leurs systèmes de fonctionnement, leurs réseaux de sens, leurs tension $(\ldots) \gg$.

La sociocritique est revendiquée et développée aussi par des auteurs comme Edmond Cros (qui s'appuie sur Althusser et Lacan) ou Pierre Zima (qui se réclame d'Adorno et de l'École de Francfort). Dans les études littéraires, l'opposition entre Pierre Barbéris et Claude 
Duchet illustre les rapports ambigus de la sociocritique et du marxisme. Au fil des années, Duchet revendique de plus en plus sa distance vis-à-vis du marxisme. Il établit de nouveaux concepts méthodologiques parmi lesquels on peut citer le sociotexte, le sociogramme, le cotexte, et le triptyque information, indice, valeur. A contrario, Barbéris (1990), tout en prônant l'intérêt de la sociocritique comme facteur de renouvellement du marxisme, n'adopte pas ces concepts, et refuse de dissocier marxisme et sociocritique.

Malgré une certaine effervescence théorique (notamment à l'université de Vincennes, autour de la revue Littérature), la sociocritique, prise entre les approches post-structuralistes et la sociologie de la littérature d'inspiration bourdieusienne, n'a pas connu de réelle institutionnalisation en France (contrairement à la Belgique, au Québec ou à la Suisse). Claude Duchet et Isabelle Tournier ont ainsi regretté que l'institution scolaire préfère aux concepts de la sociocritique des approches héritées du structuralisme « schémas unifiants à base linguistique sur des œuvres d'origine, de facture, d'histoire, de valeurs diverses » (1994). La rencontre de la sociocritique et de l'analyse du discours a permis le dépassement d'une opposition regrettable (Bordas, 2005) entre stylistique «de droite» (Sorbonne) et une sociocritique « de gauche» (Vincennes), mais au prix d'une certaine édulcoration méthodologique de la sociocritique. Ce recoupement de la sociocritique avec une autre discipline plus en vogue a achevé de la détacher du marxisme.

\section{Conclusion}

Les approches marxistes de la littérature en France sont progressivement passées d'un cadrage par le PCF à des initiatives dispersées et relativement disparates qui ont permis la diversification des circuits d'importation et des appropriations de textes marxistes. Les transformations des espaces universitaires et éditoriaux, qui avaient participé à la promotion de certaines approches de la littérature se revendiquant du marxisme, ne tardent cependant pas à réduire la place qui leur était donnée au lendemain de mai 68. Le retournement d'anciens maos au milieu des années 1970, la critique du structuralisme par certains promoteurs initiaux des théories de la littérature, la médiatisation de groupes intellectuels construits sur l'opposition au marxisme ou encore les reformulations américaines de la «théorie » et les hostilités qu'elles nourrissent contribuent à la mise à distance du matérialisme et à la retraduction de l'anticommunisme en antimarxisme dans les espaces académiques, intellectuels et littéraires. Les approches matérialistes de la littérature, plus ou moins proches de Marx et du système théorique marxiste, sont pourtant réinvesties à travers les figures fondatrices de la sociologie de 
la littérature et de la sociocritique. C'est donc toujours dans un espace conflictuel et fortement marqué par des oppositions politiques que les approches marxistes de la littérature se maintiennent péniblement à la fin $\mathrm{du} \mathrm{XX}^{\mathrm{e}}$ siècle. À la doxa qui fait du marxisme un héritage obsolète s'opposent cependant quelques initiatives plus ponctuelles. Ainsi, certains philosophes, marxistes, anciens marxistes ou apparentés (Badiou, Macherey, Rancière) se sont récemment saisis à nouveau de la question de la littérature et de son rôle politique. On peut également noter, au tournant du siècle, les rééditions de Lukács en 1999 (Balzac et le réalisme français, publié aux éditions La Découverte et préfacé par Gengembre) de Barbéris en 2000 (Le Monde de Balzac, aux éditions Kimé, avec une postface inédite), puis de Macherey en 2014 (Pour une théorie de la production littéraire, éditions de l'ÉNS Lyon, avec une postface inédite de l'auteur) qui posent chacune cette question : que peut être une approche marxiste de la littérature au $\mathrm{XXI}^{\mathrm{e}}$ siècle?

\section{Bibliographie}

Amossy, Ruth (présenté et coordonné par). 2005. Analyse du discours et sociocritique, Littérature, vol. $140, n^{\circ} 4$.

Aron, Paul, Matonti, Frédérique et Sapiro, Gisèle (dir.). 2003. Repenser le réalisme socialiste, Sociétés \& Représentations $\mathrm{n}^{\circ} 15$.

Aucouturier, Michel. 1998. Le Réalisme socialiste. Paris : Presses Universitaires de France.

Barbéris, Pierre. 1970. Balzac et le mal du siècle : contribution à une physiologie du monde moderne, Paris, Gallimard.

Barbéris, Pierre. 1990. «La sociocritique », Méthodes critiques pour l'analyse littéraire, Bordas, 151203

Barthes, Roland. 1957. Mythologies. In Oeuvres Complètes I, 671-872. Paris : Seuil (2002).

Bourdieu, Pierre. 1984. «Le champ littéraire. Préalables critiques et principes de méthode», in Lendemains, tome 9, n³6, 5-20.

Desné, Roland. 1974. «Présentation ». Histoire littéraire de la France, vol. 1. Paris : Les Editions Sociales.

Depretto, Catherine. 2010. « Le formalisme russe et ses sources. Quelques considérations de méthode. » Cahiers du monde russe 51 (4) : 565-579.

Dirkx, Paul. 2015. «Bourdieu et les études littéraires », in Bourdieu et les sciences sociales. Réception et usages, Paris, La Dispute, 161-178.

Duchet, Claude. 1971 « Pour une socio-critique, ou variation sur un incipit », in Littérature, vol1, $\mathrm{n}^{\circ} 1$, $5-14$ 
Duchet, Claude (dir.). 1979. Sociocritique, Paris, Nathan.

Duchet, Claude et Tournier Isabelle, « Sociocritique », in Béatrice Didier (dir.), Dictionnaire universel des Littératures, vol.3, 1994, 3571-3573

Ebguy, Jacques-David. 2015. « Débat critique : Pierre Barbéris aujourd'hui ? (avec les participations de B. Lyon-Caen et J. David), in Romantisme, n 168, 105-126.

Gobille, Boris. 2005. «La guerre de Change contre la "dictature structuraliste" de Tel Quel. Le "théoricisme" des avant-gardes littéraires à l'épreuve de la crise politique de mai 68 », in Raisons politiques, vol. $2, \mathrm{n}^{\circ} 18,73-96$.

Goldmann, Lucien. 2013. Le Dieu caché : Étude sur la vision tragique dans les Pensées de Pascal et dans le théâtre de Racine. Paris : Gallimard.

Jeanpierre, Laurent. 2013. « Adieux aux structures et à la dialectique. La pensée des sciences sociales aux colloques de Cerisy après Mai 1968 ».Histoire@ Politique, nº 2 :114-133.

Jdanov, Andreï. 1950. Sur la littérature, la philosophie et la musique. Paris : Les Éditions de La Nouvelle Critique.

Labica, Georges, Bensussan Gérard (dir.). 2001. Dictionnaire critique du marxisme. Paris: Presses Universitaires de France.

Martelli, Roger. 2017. Une dispute communiste. Le Comité Central d'Argenteuil sur la culture. Paris : Les Éditions Sociales.

Marx, Karl, Engels, Friedrich. 1954. Sur la littérature et l'art. Paris : Éditions sociales.

Matonti, Frédérique. 2005. Intellectuels communistes. Essai sur l'obéissance politique. La Nouvelle Critique (1967-1980). Paris : La Découverte.

Roger, Philippe. 1996. « Barthes dans les années Marx », Communications, vol. 63, nº1, 39-65.

Wurmser, André. 1950. «Chronique artistique : Réponse à Francis Jourdain ». La Pensée 32 : 127 132. 